\title{
Pruned cellular free resolutions of monomial ideals
}

\author{
Josep Àlvarez Montaner ${ }^{1}$ \\ Departament de Matemàtiques, Universitat Politècnica de Catalunya, Spain \\ Oscar Fernández-Ramos, Philippe Gimenez ${ }^{2, *}$ \\ Instituto de Investigación en Matemáticas de Valladolid (IMUVA), Universidad de Valladolid, Spain
}

\begin{abstract}
Using discrete Morse theory, we give an algorithm that prunes the excess of information in the Taylor resolution and constructs a new cellular free resolution for an arbitrary monomial ideal. The pruned resolution is not simplicial in general, but we can slightly modify our algorithm in order to obtain a simplicial resolution. We also show that the Lyubeznik resolution fits into our pruning strategy. The pruned resolution is not always minimal but it is a lot closer to the minimal resolution than the Taylor and the Lyubeznik resolutions as we will see in some examples. We finally use our methods to give a different approach to the theory of splitting of monomial ideals. We deduce from this splitting strategy that the pruned resolution is always minimal in the case of edge ideals of paths and cycles.
\end{abstract}

Keywords: free resolution, monomial ideal, discrete Morse theory, Betti splitting 2010 MSC: 13D45, 13N10

\section{Introduction}

Let $R=\mathbb{k}\left[x_{1}, \ldots, x_{n}\right]$ be the polynomial ring over a field $\mathbb{k}$ and $I \subseteq R$ a monomial ideal. The study of minimal free resolutions of these ideals has been a very active area of research during the last decades. There are topological and combinatorial formulae, as those of Hochster [14] or Gasharov, Peeva and Welker [11, to describe their multigraded Betti numbers but, except for some specific classes of monomial ideals (see, e.g., 8], [5] or [18]), the problem of describing a minimal multigraded free resolution explicitly has shown to be difficult.

Another strategy is to study non-minimal free resolutions. These reveal less information than minimal free resolutions do but are often much easier to describe. The most significant ones, that we will also comment on in this paper, are the Taylor resolution [21] and the Lyubeznik resolution [17, but one should also mention the Scarf resolution of arbitrary monomial ideals obtained by deformation of exponents [5] and the hull resolution [6. An interesting feature of the Taylor and the Lyubeznik resolutions is that they fit in the theory of simplicial resolutions introduced by Bayer, Peeva and Sturmfels in [5] and further extended to regular cellular resolutions and CW-resolutions in [6] and [16] respectively. The idea behind these three concepts is to associate to a free resolution of a monomial ideal a simplicial complex

\footnotetext{
${ }^{*}$ Corresponding author

Email addresses: Josep.Alvarez@upc.es (Josep Àlvarez Montaner), pgimenez@agt.uva.es (Philippe Gimenez)

${ }^{1}$ Partially supported by the Generalitat de Catalunya grant SGR2017-932 and the Spanish Ministerio de Economía y Competitividad grant MTM2015-69135-P. He is a member of the Barcelona Graduate School of Mathematics (BGSMath).

${ }^{2}$ Partially supported by the Spanish Ministerio de Economia y Competitividad grant MTM2016-78881-P and Consejería de Educación de la Junta de Castilla y León grant VA128G18.
} 
(respectively a regular cell complex, a CW-complex) that carries in its structure the algebraic structure of the free resolution. It is worth pointing out that Velasco proved in 23 that there exist monomial ideals whose minimal free resolutions cannot be described by a CW-complex.

By adapting the discrete Morse theory developed by Forman [9] and Chari [7, Batzies and Welker provided in 4] a method to reduce a given regular cellular resolution. In particular, they proved that the Lyubeznik resolution can be obtained in this way from the Taylor resolution. Let's point out that discrete Morse theory has the inconvenient fact that it can't be used iteratively. To overcome this issue, one can use the algebraic discrete Morse theory developed independently by Sköldberg [20] and Jöllenbeck and Welker [16. In this work, we use a similar strategy to reduce the Taylor resolution and obtain cellular and simplicial free resolutions that are closer to the minimal one than the Lyubeznik resolution. Essentially, the information given by the Taylor resolution can be encoded in a directed graph and the obstruction to its minimality can be observed in some of the edges of this graph. What we will do is to remove, in a convenient order, some of these edges to provide a smaller resolution. In some sense, we are pruning the excess of information given by the Taylor resolution in a simple and efficient way.

The organization of this paper is as follows. In Section 2 , we review the notion of cellular resolution and introduce the basics on discrete Morse theory that will be needed throughout this work. In Section 3. we present our main results. We first provide an algorithm (Algorithm 3.1) that, starting from the Taylor resolution of a monomial ideal, allows to construct a smaller cellular free resolution (Theorem 3.4). The resolution that we obtain is not simplicial in general, but we can adapt our pruning algorithm to produce a simplicial free resolution (Algorithm 3.7). Indeed, the Lyubeznik resolution fits into this pruning strategy as shown in Algorithm 3.9. Other variants of our method are also mentioned.

In Section 4, we illustrate our results with several examples. We implemented our algorithms using CoCoALib [1] for constructing pruned resolutions in the non-trivial examples contained in this section. Finally, in Section 5 we present a connection between our method and the theory of Betti splittings introduced by Eliahou and Kervaire [8] and later developed by Francisco, Hà and Van Tuyl [10]. We provide a sufficient condition for having a Betti splitting by checking some prunings in our algorithm. We use this approach to prove that the pruned resolution is minimal for edge ideals associated to paths and cycles.

\section{Cellular resolutions using discrete Morse theory}

Let $R=\mathbb{k}\left[x_{1}, \ldots, x_{n}\right]$ be the polynomial ring in $n$ variables with coefficients in a field $\mathbb{k}$. An ideal $I \subseteq R$ is monomial if it may be generated by monomials $\mathbf{x}^{\alpha}:=x_{1}^{\alpha_{1}} \cdots x_{n}^{\alpha_{n}}$, where $\alpha \in \mathbb{Z}_{\geq 0}^{n}$. As usual, we set $|\alpha|:=\alpha_{1}+\cdots+\alpha_{n}$. Moreover, given a monomial set of generators of an ideal $I,\left\{m_{1}, \ldots, m_{r}\right\}$, we will consider the monomials $m_{\sigma}:=\operatorname{lcm}\left(m_{i} \mid \sigma_{i}=1\right)$ for any $\sigma \in\{0,1\}^{r}$. Denote by $\varepsilon_{1}, \ldots, \varepsilon_{r}$ the standard basis of $\mathbb{Z}^{r}$.

A $\mathbb{Z}^{n}$-graded free resolution of $R / I$ is an exact sequence of free $\mathbb{Z}^{n}$-graded modules:

$$
\mathbb{F}_{\bullet}: \quad 0 \longrightarrow F_{p} \stackrel{d_{n}}{\longrightarrow} \cdots \longrightarrow F_{1} \stackrel{d_{1}}{\longrightarrow} F_{0} \longrightarrow R / I \longrightarrow 0
$$

where the $i$-th term is of the form

$$
F_{i}=\bigoplus_{\alpha \in \mathbb{Z}^{n}} R(-\alpha)^{\beta_{i, \alpha}}
$$

We say that $\mathbb{F} \bullet$ is minimal if the matrices of the homogeneous morphisms $d_{i}: F_{i} \longrightarrow F_{i-1}$ do not contain invertible elements. In this case, the exponents $\beta_{i, \alpha}$ form a set of invariants of $R / I$ known as its multigraded Betti numbers. Throughout this work, we will mainly consider the coarser $\mathbb{Z}$-graded free 
resolution. In this case, we will encode the $\mathbb{Z}$-graded Betti numbers in the so-called Betti diagram of $R / I$ where the entry on the $i$ th column and $j$ th row of the table is $\beta_{i, i+j}$ :

$\begin{array}{ccccc} & 0 & 1 & 2 & \cdots \\ \text { total: } & \cdot & \cdot & \cdot & \\ 0: & \beta_{0,0} & \beta_{1,1} & \beta_{2,2} & \cdots \\ 1: & \beta_{0,1} & \beta_{1,2} & \beta_{2,3} & \cdots \\ \vdots & \vdots & \vdots & \vdots & \end{array}$

\subsection{Cellular resolutions}

A CW-complex $X$ is a topological space obtained by attaching cells of increasing dimensions to a discrete set of points $X^{(0)}$. Let $X^{(i)}$ denote the set of $i$-cells of $X$ and consider the set of all cells $X^{(*)}:=\bigcup_{i>0} X^{(i)}$. Then, we can view $X^{(*)}$ as a poset with the partial order given by $\sigma^{\prime} \leq \sigma$ if and only if $\sigma^{\prime}$ is contained in the closure of $\sigma$. We can also give a $\mathbb{Z}^{n}$-graded structure to $X$ by means of an order preserving map $g r: X^{(*)} \longrightarrow \mathbb{Z}_{\geq 0}^{n}$.

We say that the free resolution (2.1) is cellular (or is a $C W$-resolution) if there exists a $\mathbb{Z}^{n}$-graded CW-complex $(X, g r)$ such that, for all $i \geq 1$ :

- there exists a basis $\left\{e_{\sigma}\right\}$ of $F_{i}$ indexed by the $(i-1)$-cells of $X$, such that if $e_{\sigma} \in R(-\alpha)^{\beta_{i, \alpha}}$ then $\operatorname{gr}(\sigma)=\alpha$, and

- the differential $d_{i}: F_{i} \longrightarrow F_{i-1}$ is given by

$$
e_{\sigma} \mapsto \sum_{\sigma \geq \sigma^{\prime} \in X^{(i-1)}}\left[\sigma: \sigma^{\prime}\right] \mathbf{x}^{g r(\sigma)-g r\left(\sigma^{\prime}\right)} e_{\sigma^{\prime}}, \quad \forall \sigma \in X^{(i)}
$$

where $\left[\sigma: \sigma^{\prime}\right]$ denotes the coefficient of $\sigma^{\prime}$ in the image of $\sigma$ by the differential map in the cellular homology of $X$.

In what follows, whenever we want to emphasize such a cellular structure, we will denote the free resolution as $\mathbb{F}_{\bullet}=\mathbb{F}_{\bullet}^{(X, g r)}$. If $X$ is a simplicial complex, we say that the free resolution is simplicial. This is the case for the following two well-known examples.

- The Taylor resolution: The most recurrent example of simplicial free resolution is the Taylor resolution discovered in [21]. Using the above terminology, we can describe it as follows. Let $I=$ $\left\langle m_{1}, \ldots, m_{r}\right\rangle \subseteq R$ be a monomial ideal. Consider the full simplicial complex on $r$ vertices, $X_{\text {Taylor }}$, whose faces are labelled by $\sigma \in\{0,1\}^{r}$ or, equivalently, by the corresponding monomials $m_{\sigma}$. We have a natural $\mathbb{Z}^{n}$-grading on $X_{\text {Taylor }}$ by assigning $\operatorname{gr}(\sigma)=\alpha \in \mathbb{Z}^{n}$ where $\mathbf{x}^{\alpha}=m_{\sigma}$. The Taylor resolution is the simplicial resolution $\mathbb{F}^{\left(X_{\text {Taylor }}, g r\right)}$.

- The Lyubeznik resolution: Another important example of simplicial resolution is the one considered by Lyubeznik in [17. Let's s start fixing an order $m_{1} \leq \cdots \leq m_{r}$ on a generating set of a monomial ideal $I \subseteq R$. Consider the simplicial subcomplex $X_{\text {Lyub }} \subseteq X_{\text {Taylor }}$ whose faces of dimension $s$ are labelled by those $\sigma=\varepsilon_{i_{0}}+\cdots+\varepsilon_{i_{s}} \in\{0,1\}^{r}$ with $i_{0}<\cdots<i_{s}$ such that, for all $t<s$ and all $j<i_{t}$,

$$
m_{j} \backslash \operatorname{lcm}\left(m_{i_{t}}, \ldots, m_{i_{s}}\right) .
$$

The Lyubeznik resolution is the simplicial resolution $\mathbb{F}_{\bullet}^{\left(X_{\text {Lyub }}, g r\right)}$. 


\subsection{Discrete Morse theory}

Forman introduced in 9 the discrete Morse theory as a method to reduce the number of cells in a CW-complex without changing its homotopy type. Batzies and Welker adapted this technique in [4] to the study of cellular resolutions; see also [24. Indeed, they used the reformulation of discrete Morse theory in terms of acyclic matchings given by Chari in [7] in order to obtain, given a regular cellular resolution (most notably the Taylor resolution), a reduced cellular resolution.

This is also our approach in this work. Let's start recalling from [4 the preliminaries on discrete Morse theory. Consider the directed graph $G_{X}$ on the set of cells of a regular $\mathbb{Z}^{n}$-graded $\mathrm{CW}$-complex $(X, g r)$ which edges are given by

$$
E_{X}=\left\{\sigma \longrightarrow \sigma^{\prime} \mid \sigma^{\prime} \leq \sigma, \operatorname{dim} \sigma^{\prime}=\operatorname{dim} \sigma-1\right\} .
$$

For a given set of edges $\mathcal{A} \subseteq E_{X}$, denote by $G_{X}^{\mathcal{A}}$ the graph obtained by reversing the direction of the edges in $\mathcal{A}$, i.e., the directed graph with edge $\mathrm{S}^{3}$

$$
E_{X}^{\mathcal{A}}=\left(E_{X} \backslash \mathcal{A}\right) \cup\left\{\sigma^{\prime} \Longrightarrow \sigma \mid \sigma \longrightarrow \sigma^{\prime} \in \mathcal{A}\right\} .
$$

When each cell of $X$ occurs in at most one edge of $\mathcal{A}$, we say that $\mathcal{A}$ is a matching on $X$. A matching $\mathcal{A}$ is acyclic if the associated graph $G_{X}^{\mathcal{A}}$ is acyclic, i.e., does not contain any directed cycle. Given an acyclic matching $\mathcal{A}$ on $X$, the $\mathcal{A}$-critical cells of $X$ are the cells of $X$ that are not contained in any edge of $\mathcal{A}$. Finally, an acyclic matching $\mathcal{A}$ is homogeneous whenever $\operatorname{gr}(\sigma)=\operatorname{gr}\left(\sigma^{\prime}\right)$ for any edge $\sigma \longrightarrow \sigma^{\prime} \in \mathcal{A}$.

Proposition 2.1 (4, Proposition 1.2]). Let $(X$, gr $)$ be a regular $\mathbb{Z}^{n}$-graded $C W$-complex and $\mathcal{A}$ a homogeneous acyclic matching. Then, there is a (not necessarily regular) $C W$-complex $X_{\mathcal{A}}$ whose $i$-cells are in one-to-one correspondence with the $\mathcal{A}$-critical $i$-cells of $X$, such that $X_{\mathcal{A}}$ is homotopically equivalent to $X$, and that inherits the $\mathbb{Z}^{n}$-graded structure.

In the theory of cellular resolutions, we have the following consequence.

Theorem 2.2 ([4, Theorem 1.3]). Let $I \subseteq R=\mathbb{k}\left[x_{1}, \ldots, x_{n}\right]$ be a monomial ideal. Assume that $(X, g r)$ is a regular $\mathbb{Z}^{n}$-graded $C W$-complex that defines a cellular resolution $\mathbb{F}_{\bullet}^{(X, g r)}$ of $R / I$. Then, for a homogeneous acyclic matching $\mathcal{A}$ on $G_{X}$, the $\mathbb{Z}^{n}$-graded $C W$-complex $\left(X_{\mathcal{A}}, g r\right)$ supports a cellular resolution $\mathbb{F}_{\bullet}^{\left(X_{\mathcal{A}}, g r\right)}$ of $R / I$.

The differentials of the cellular resolution $\mathbb{F}_{\bullet}^{\left(X_{\mathcal{A}}, g r\right)}$ can be explicitely described in terms of the differentials of $\mathbb{F}^{(X, g r)}$ (see [4, Lemma 7.7]) as we will see later in Example 3.2. Since in this paper we are mainly concerned by the Betti numbers, we will not write down the differentials in general.

\subsection{Algebraic discrete Morse theory}

One of the main inconveniences of discrete Morse theory is that the CW-complex $\left(X_{\mathcal{A}}, g r\right)$ that we obtain for a given homogeneous acyclic matching $\mathcal{A}$ is not necessarily regular. Therefore, we cannot always iterate the procedure. To overcome such an obstacle, one may use algebraic discrete Morse theory developed independently by Sköldberg [20] and Jöllenbeck and Welker [16].

This approach works directly with an initial free resolution $\mathbb{F}$. without paying attention whether it has a cellular structure or not. Given a basis $X=\bigcup_{i \geq 0} X^{(i)}$ of the corresponding free modules $F_{i}$, we may consider the directed graph $G_{X}$ on the set of basis elements with the corresponding set of edges $E_{X}$. Then, we may follow the same constructions as in the previous subsection. Namely, we may define

\footnotetext{
${ }^{3}$ For the sake of clarity, the arrows that we reverse will be denoted by $\Longrightarrow$.
} 
an acyclic matching $\mathcal{A} \subseteq E_{X}$ (see [16, Definition 2.1]) but, in this case, we have to make sure that the coefficient $\left[\sigma: \sigma^{\prime}\right]$ in the differential corresponding to an edge $\sigma \longrightarrow \sigma^{\prime} \in \mathcal{A}$ is a unit. We consider the $\mathcal{A}$-critical basis elements $X_{\mathcal{A}}$ and construct a free resolution $\mathbb{F}_{\bullet}^{X_{\mathcal{A}}}$ that is homotopically equivalent to $\mathbb{F}$. (see [16, Theorem 2.2]).

\section{Pruning the Taylor resolution}

In [4, the Lyubeznik resolution is obtained from the Taylor resolution through discrete Morse theory by detecting a suitable homogeneous acyclic matching $\mathcal{A}$ on the simplicial complex $X_{\text {Taylor }}$ such that $X_{\text {Lyub }}=X_{\mathcal{A}}$. In this section, we use a similar approach to provide some new cellular free resolutions for monomial ideals. We should point out that the framework considered in [4] is slightly more general. To keep notation as simple as possible, we decided to stick to the case of monomial ideals in a polynomial ring.

\subsection{A cellular free resolution}

Let $I=\left\langle m_{1}, \ldots, m_{r}\right\rangle \subseteq R$ be a monomial ideal. Our starting point is the Taylor resolution $\mathbb{F}_{\bullet}^{\left(X_{\text {Taylor }}, g r\right)}$. This resolution is, in general, far from being minimal. In other words, the directed graph $G_{X_{\text {Taylor }}}$ associated to $X_{\text {Taylor }}$ contains a lot of unnecessary information. Our goal is to prune this excess of information in a very simple way. More precisely, we give an algorithm that produces, at each step, a homogeneous acyclic matching on $X_{\text {Taylor }}$. Using discrete Morse theory, this will provide a cellular free resolution of $R / I$. It will not be minimal in general, but it will be smaller than the Lyubeznik resolution.

Algorithm 3.1. (Pruned resolution)

INPUT: The set of edges $E_{X_{\text {Taylor }}}$.

For $j$ from 1 to $r$, incrementing by 1

(j) Prune the edge $\sigma+\varepsilon_{j} \longrightarrow \sigma$ for all $\sigma \in\{0,1\}^{r}$ such that $\sigma_{j}=0$, where 'prune' means remove the edg $€^{4}$ if it survived after step $(j-1)$ and $\operatorname{gr}(\sigma)=\operatorname{gr}\left(\sigma+\varepsilon_{j}\right)$.

Return: The set $\mathcal{A}_{P}$ of edges that have been pruned.

Example 3.2. When $r=3$, we can visualize the steps of the algorithm over the directed graph as follows:
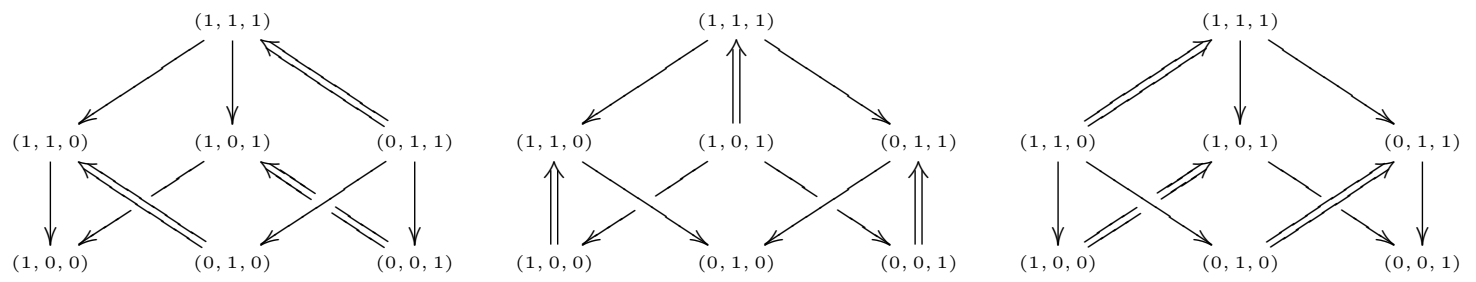

The double arrows indicate the direction of the pruning step, that is, the arrows that will be pruned at each step if the degree of their two vertices coincide (and if they have not been pruned at a previous step).

\footnotetext{
${ }^{4}$ When we remove an edge, we also remove its two vertices and all the edges passing through these two vertices.
} 
Let's illustrate how our algorithm works with an easy example: the edge ideal associated to the 3 -cycle, $I=\left\langle x_{1} x_{2}, x_{2} x_{3}, x_{1} x_{3}\right\rangle \subset R=\mathbb{k}\left[x_{1}, x_{2}, x_{3}\right]$. The Taylor resolution of $I$ is

$$
0 \rightarrow R \stackrel{\left(\begin{array}{c}
1 \\
-1 \\
1
\end{array}\right)}{\longrightarrow} R^{3} \stackrel{\left(\begin{array}{ccc}
x_{3} & x_{3} & 0 \\
-x_{1} & 0 & x_{1} \\
0 & -x_{2} & -x_{2}
\end{array}\right)}{\longrightarrow} R^{3} \stackrel{\left(x_{1} x_{2} \quad x_{2} x_{3} \quad x_{1} x_{3}\right)}{\longrightarrow} I \rightarrow 0 \quad .
$$

The directed graph $G_{X_{\text {Taylor }}}$ associated to this resolution is

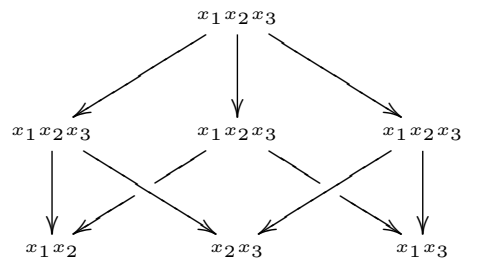

where, in order to identify clearly which edges can be pruned, we label the vertices by the monomial $m_{\sigma}$ instead of $\sigma$. At the first step of the algorithm we prune the arrow $(0,1,1) \Rightarrow(1,1,1)$ as indicated in the first graph below. Once we remove this edge, we observe on the second graph that no more arrows can be pruned at the next two steps.
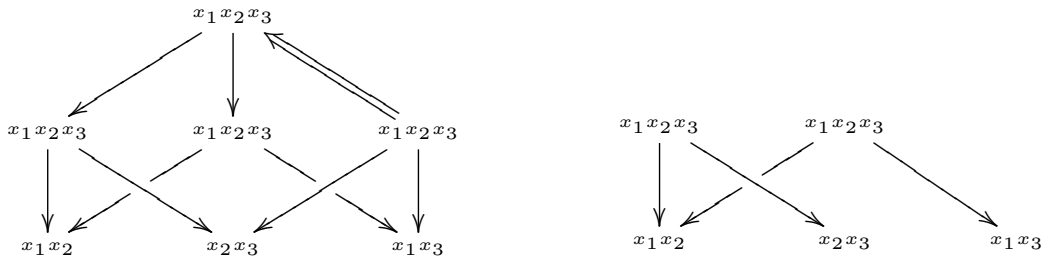

The pruned resolution, which is minimal in this case, is as follows:

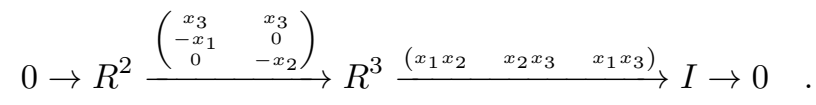

The main result in this section is Theorem 3.4 where we show that the output $\mathcal{A}_{P}$ of Algorithm 3.1 is a homogeneous matching such that $\left(X_{\mathcal{A}_{P}}, g r\right)$ supports a cellular free resolution of $R / I$. In order to do so, we will first prove that each step of Algorithm 3.1. produces a homogeneous acyclic matching. In other words, pruning in a fixed direction gives a new smaller free resolution.

Proposition 3.3. Let $E \subseteq E_{X_{\text {Taylor }}}$ be a subset of edges and $\mathcal{A}_{j} \subseteq E$ be the set of pruned edges in the direction of $\varepsilon_{j}$. Namely, $\mathcal{A}_{j}$ are the edges $\sigma+\varepsilon_{j} \longrightarrow \sigma$ in $E$ such that $\sigma_{j}=0$ and $\operatorname{gr}(\sigma)=\operatorname{gr}\left(\sigma+\varepsilon_{j}\right)$. Then $\mathcal{A}_{j}$ is a homogeneous acyclic matching.

Proof. In the graph $G_{X_{\text {Taylor }}}$, every vertex $\sigma^{\prime}$ is involved in exactly one edge of the form $\sigma+\varepsilon_{j} \longrightarrow \sigma: \sigma^{\prime}=\sigma$ if $\sigma_{j}^{\prime}=0$ and $\sigma^{\prime}=\sigma+\varepsilon_{j}$ otherwise. Thus, $\mathcal{A}_{j}$ is a matching. It is acyclic since we are only reversing arrows in a fixed direction and it is impossible to construct a cycle in $G_{X_{\text {Taylor }}}$ with this restriction. It is homogeneous by construction of $\mathcal{A}_{j}$ in our pruning step.

As a direct consequence, we get our desired cellular free resolution.

Theorem 3.4. Let $I \subseteq R=\mathbb{k}\left[x_{1}, \ldots, x_{n}\right]$ be a monomial ideal and $\mathcal{A}_{P} \subseteq E_{X_{\text {Taylor }}}$ be the set of pruned edges obtained using Algorithm 3.1. Then, the $\mathbb{Z}^{n}$-graded $C W$-complex $\left(X_{\mathcal{A}_{P}}\right.$, gr $)$ supports a cellular free resolution $\mathbb{F}_{\bullet}^{\left(X_{\left.\mathcal{A}_{P}, g r\right)}\right.}$ of $R / I$. 
Remark 3.5. The free resolution $\mathbb{F}_{\bullet}^{\left(X_{\mathcal{A}_{P}}, g r\right)}$, like the Lyubeznik resolution, strongly depends on the order of the generators of the monomial ideal $I$. In general, it is neither simplicial (while the Lyubeznik resolution is always simplicial) nor minimal.

A nice feature about the pruning algorithm is that we do not need to care if the original system of generators of the monomial ideal is minimal or not. Roughly speaking, the pruning algorithm will always remove the excess of information given by the extra generators.

Lemma 3.6. Let $I=\left\langle m_{1}, \ldots, m_{s}\right\rangle \subseteq R$ be a monomial ideal, and let $\left\{m_{i_{1}}, \ldots, m_{i_{r}}\right\}$ be its minimal set of generators with $1 \leq i_{1}<\cdots<i_{r} \leq s$. Let $X_{s}$ and $X_{r}$ be the Taylor simplicial complexes associated to these two sets of generator $\$$ and $\mathcal{A}_{P}^{s} \subseteq E_{X_{s}}, \mathcal{A}_{P}^{r} \subseteq E_{X_{r}}$ be the sets of pruned edges obtained by applying Algorithm 3.1 to each case. Then, there is an isomorphism of $\mathbb{Z}^{n}$-graded $C W$ complexes $\left(X_{\mathcal{A}_{P}^{r}}, g r\right) \cong\left(X_{\mathcal{A}_{P}^{s}}, g r\right)$. In particular, both sets of generators lead to the same cellular free resolution of $R / I$ in Theorem 3.4 .

Proof. Assume that $m_{j}$ is a generator of $I$ that does not belong to the minimal generating set $\left\{m_{i_{1}}, \ldots, m_{i_{r}}\right\}$. We want to check that all the vertices $\sigma=\left(\sigma_{1}, \ldots, \sigma_{s}\right)$ with $\sigma_{j}=1$ in the graph associated to the Taylor complex $X_{s}$ are pruned using Algorithm 3.1 .

There exists a minimal generator $m_{i_{k}}$ such that $m_{i_{k}} \mid m_{j}$. Therefore, at step $\left(i_{k}\right)$ of Algorithm 3.1, we must prune all the edges $\sigma+\varepsilon_{i_{k}} \longrightarrow \sigma$ with $\sigma_{j}=1$ and $\sigma_{i_{k}}=0$ that have survived in the previous steps. In the case that the edge $\sigma+\varepsilon_{\ell}+\varepsilon_{i_{k}} \longrightarrow \sigma+\varepsilon_{i_{k}}$ has been pruned at a previous step $(\ell)$ of the algorithm, then $\operatorname{gr}\left(\sigma+\varepsilon_{\ell}+\varepsilon_{i_{k}}\right)=\operatorname{gr}\left(\sigma+\varepsilon_{i_{k}}\right)=\operatorname{gr}(\sigma)$ and thus $\operatorname{gr}(\sigma)=\operatorname{gr}\left(\sigma+\varepsilon_{\ell}\right)$ so the edge $\sigma+\varepsilon_{\ell} \longrightarrow \sigma$ has to be pruned at the same step $(\ell)$. In particular neither the vertex $\sigma$ nor the vertex $\sigma+\varepsilon_{i_{k}}$ survive at this previous step $(\ell)$ of the algorithm.

\subsection{A simplicial free resolution}

If $\mathcal{A}_{P}$ is the output of Algorithm 3.1, the cellular complex $X_{\mathcal{A}_{P}}$ may not be simplicial, that is, it may not satisfy the property that given $\tau \in X_{\mathcal{A}_{P}}$, then $\sigma \in X_{\mathcal{A}_{P}}$ for any $\sigma \leq \tau$. In other words, the pruned free resolution $\mathbb{F}_{\bullet}^{\left(X_{\mathcal{A}_{P}}, g r\right)}$ obtained in Theorem 3.4 is always cellular but it may not be simplicial. If we choose carefully the edges that we prune in Algorithm 3.1 in order to preserve this property, we will obtain a simplicial free resolution of $R / I$ that will be bigger, in general, than the one in Theorem 3.4 .

Algorithm 3.7. (Simplicial pruned resolution)

INPUT: The set of edges $E_{X_{\text {Taylor }}}$.

For $j$ from 1 to $r$, incrementing by 1

(j) Prune the edge $\sigma \longrightarrow \sigma+\varepsilon_{j}$ for all $\sigma \in\{0,1\}^{r}$ such that $\sigma_{j}=0$, where 'prune' means remove the edge if it survived after step $(j-1), \operatorname{gr}(\sigma)=\operatorname{gr}\left(\sigma+\varepsilon_{j}\right)$ and no face $\tau>\sigma$ survives at this step $(j)$.

REturn: The set $\mathcal{A}_{S}$ of edges that have been pruned.

Using the same idea as in Proposition 3.3, we have that each step of Algorithm 3.7 produces a homogeneous acyclic matching on $X_{\text {Taylor }}$ and hence, the free resolution $\mathbb{F}_{\bullet}^{\left(X_{\mathcal{A}_{S}}, g r\right)}$ is a simplicial free resolution of the monomial ideal $I$ if $\mathcal{A}_{S}$ is the output of Algorithm 3.7 .

Remark 3.8. It could be the case that the simplicial pruned resolution that we obtain with Algorithm 3.7 may admit further refinement. This happens when an edge $\sigma+\varepsilon_{j} \longrightarrow \sigma$, that would be pruned at step

\footnotetext{
${ }^{5}$ We have that $X_{r}$ is a subcomplex of $X_{s}$
} 
$(j)$ of Algorithm 3.1. may not be pruned at step $(j)$ of Algorithm 3.7 because there is a face $\tau>\sigma$ that survives at this step but this face $\tau$ is pruned in a posterior step. In this case we may apply Algorithm 3.7 once again, with the set of edges $E_{X_{\mathcal{A}_{S}}}$ as input instead of $E_{X_{\text {Taylor }}}$, in order to prune the edge $\sigma+\varepsilon_{j} \longrightarrow \sigma$. Indeed we may repeat Algorithm 3.7 until we have nothing left to prune. We will observe this phenomenon later in Example 4.1 contructing the simplicial pruned resolution of the 5-cycle.

\subsection{The Lyubeznik resolution revisited}

The Lyubeznik resolution can be also obtained from the Taylor resolution using our pruning algorithm. In this case, the edges of the Taylor complex $X_{\text {Taylor }}$ that we prune are obtained using the following:

Algorithm 3.9. (The Lyubeznik resolution via pruning)

INPUT: The set of edges $E_{X_{\text {Taylor }}}$.

For $j$ from 1 to $r$, incrementing by 1

(j) Prune the edge $\sigma \longrightarrow \sigma+\varepsilon_{j}$ for all $\sigma \in\{0,1\}^{r}$ such that $\sigma_{i}=0$ for all $i \leq j$, where 'prune' means remove the edge if it survived after step $(j-1)$ and $\operatorname{gr}(\sigma)=\operatorname{gr}\left(\sigma+\varepsilon_{j}\right)$.

RETURN: The set $\mathcal{A}_{L}$ of edges that have been pruned.

As in Proposition 3.3 , each step of Algorithm 3.9 produces a homogeneous acyclic matching on $X_{\text {Taylor }}$ and since $X_{\mathcal{A}_{L}}=X_{\text {Lyub }}$, this provides a simple proof of the result in [4, §3] that shows that the Lyubeznik resolution can be obtained using discrete Morse theory. We also have that the Lyubeznik resolution is simplicial because the pruning that we consider in Algorithm 3.9, as the one in Algorithm 3.7, preserves the simplicial property. In this sense, we may understand the pruned resolution given in Theorem 3.4 and the simplicial pruned resolution given in Subsection 3.2, as refinements of the Lyubeznik resolution. Finally, we mention that generalizations of Lyubeznik resolutions have been studied by Novik in [19] using a completely different approach.

\subsection{Some variants of the pruning algorithm}

The methods developed in this work can be extended in several different directions. The aim of this subsection is to present some of them.

- General setup: Batzies and Welker [4 use a slightly more general framework for discrete Morse theory than the one considered in this paper. The interested reader should be able to adapt our pruning algorithm to their framework.

- Partial pruning: Notice that the acyclic matchings considered in Algorithms 3.1, 3.7 and 3.9 satisfy $\mathcal{A}_{P} \supseteq \mathcal{A}_{S} \supseteq \mathcal{A}_{L}$. One may also consider any convenient subset $\mathcal{A}_{P} \supseteq \mathcal{A}^{\prime}$ of pruning edges. Indeed, we may consider many different variants using algebraic discrete Morse theory iteratively. We only have to pick a convenient edge at each iteration.

- Pruning other resolutions: The method that we present here always starts with the Taylor resolution but we may start with other non-minimal free resolutions, the Lyubeznik resolution for example. The advantage of the Taylor resolution is that it does not depend on the order of the generators, and the simplicity of its construction makes our algorithm very easy to present and implement.

$\cdot \nu$-invariants: A new set of invariants that measure the acyclicity of the linear strands of a minimal free resolution of a graded ideal was introduced in 2. We may obtain an approximation to these invariants by applying the following iteration of the pruning algorithm. We first apply Algorithm 3.1 to obtain the CW-complex $X_{\mathcal{A}_{P}}$ and its corresponding free resolution. Then, we apply the pruning algorithm to $X_{\mathcal{A}_{P}}$ with the following variant: 
(j) Prune the edge $\sigma+\varepsilon_{j} \longrightarrow \sigma$ for all $\sigma \in\{0,1\}^{r}$ such that $\sigma_{j}=0$, where 'prune' means remove the edge if it survived after step $(j-1)$ and $\operatorname{gr}(\sigma)=\operatorname{gr}\left(\sigma+\varepsilon_{j}\right)-1$. Here $\operatorname{gr}(\sigma)=|\alpha| \in \mathbb{Z}$ where $\mathbf{x}^{\alpha}=m_{\sigma}$.

\section{Examples}

In this section, we will illustrate that the pruned free resolution described in Section 3.1 is fairly close to the minimal free resolution in some examples. Indeed, we will compare the pruned resolution to the simplicial free resolution obtained in Section 3.2 and to the Lyubeznik resolution by means of their corresponding Betti diagram.

\subsection{First examples}

Already for some simple examples we can appreciate the better behavior of the pruned resolutions with respect to the Lyubeznik resolution.

Example 4.1. We are going to describe the steps of the pruning Algorithms described in Section 3 for the edge ideals associated to a 5 -path and a 5-cycle:

- Let $I=\left\langle x_{1} x_{2}, x_{2} x_{3}, x_{3} x_{4}, x_{4} x_{5}\right\rangle$ be the edge ideal of a 5 -path. The steps performed with Algorithm 3.1 are the following:

- Step (1): No edge is pruned.

- Step (2): We prune the edges $(1,0,1,0) \Rightarrow(1,1,1,0)$ and $(1,0,1,1) \Rightarrow(1,1,1,1)$.

- Step (3): We prune the edge $(0,1,0,1) \Rightarrow(0,1,1,1)$.

. Step (4): No edge is pruned.

The two edges pruned in Step (2) are also pruned using Algorithm 3.7 but are not pruned using Algorithm 3.9. The edge pruned in Step (3) is not pruned neither using Algorithm 3.7 nor Algorithm 3.9. Therefore, the Betti diagrams of the pruned, the simplicial pruned, and the Lyubeznik resolutions are respectively:

$\begin{array}{ccccc} & 0 & 1 & 2 & 3 \\ \text { total: } & 1 & 4 & 4 & 1 \\ 0: & 1 & . & . & . \\ 1: & . & 4 & 3 & . \\ 2: & . & . & 1 & 1\end{array}$

$\begin{array}{ccccc} & 0 & 1 & 2 & 3 \\ \text { total: } & 1 & 4 & 5 & 2 \\ 0: & 1 & . & . & . \\ 1: & . & 4 & 3 & 1 \\ 2: & . & . & 2 & 1\end{array}$

$\begin{array}{cccccc} & 0 & 1 & 2 & 3 & 4 \\ \text { total: } & 1 & 4 & 6 & 4 & 1 \\ 0: & 1 & . & . & . & . \\ \text { 1: } & . & 4 & 3 & 2 & 1 \\ 2: & . & . & 3 & 2 & .\end{array}$

The pruned resolution is minimal. The simplicial pruned resolution is not minimal but it is as small as possible since the ideal $I$ has no minimal simplicial resolution as one can check using an argument similar to the one used in [15, Section 3.2] for the 4-cycle. The Lyubeznik resolution coincides with the Taylor resolution since no edge has been pruned through Algorithm 3.9 .

- Let $I=\left\langle x_{1} x_{2}, x_{2} x_{3}, x_{3} x_{4}, x_{4} x_{5}, x_{5} x_{1}\right\rangle$ be the edge ideal of a 5 -cycle. The steps performed with Algorithm 3.1 are the following:

- Step (1): We prune four edges $(0,1,0,0,1) \Rightarrow(1,1,0,0,1),(0,1,1,0,1) \Rightarrow(1,1,1,0,1),(0,1,0,1,1) \Rightarrow$ $(1,1,0,1,1)$ and $(0,1,1,1,1) \Rightarrow(1,1,1,1,1)$.

. Step (2): We prune $(1,0,1,0,0) \Rightarrow(1,1,1,0,0)$ and $(1,0,1,1,0) \Rightarrow(1,1,1,1,0)$.

. Step (3): We prune the edge $(0,1,0,1,0) \Rightarrow(0,1,1,1,0)$.

Step (4): We prune $(0,0,1,0,1) \Rightarrow(0,0,1,1,1)$ and $(1,0,1,0,1) \Rightarrow(1,0,1,1,1)$.

- Step (5): We prune the edge $(1,0,0,1,0) \Rightarrow(1,0,0,1,1)$.

The edges pruned at Step (1) are also pruned using Algorithm 3.7 and Algorithm 3.9. The two edges pruned at Step (2) can not be pruned using Algorithm 3.7 because the vertices $(1,0,1,0,1)$ and 
$(1,0,1,1,1)$ remain at this step of the algorithm. The edges pruned at Step (3) and (4) are also pruned in Algorithm 3.7. The edge pruned at Step (5) is not pruned in Algorithm 3.7. Notice that the two edges that prohibited the pruning at Step (3) of Algorithm 3.7 were pruned at Step (4). This means that we can perform another round of Algorithm 3.7 that will prune the two edges that could not be pruned before at step (2) as observed in Remark 3.8 .

On the other hand, note that after the first step, no more edge can be pruned through Algorithm 3.9. We thus obtain that the Betti diagrams of the pruned, the simplicial pruned, and the Lyubeznik resolutions are the following respectively:

$\begin{array}{ccccc} & 0 & 1 & 2 & 3 \\ \text { total: } & 1 & 5 & 5 & 1 \\ 0: & 1 & . & . & . \\ 1: & . & 5 & 5 & . \\ 2: & . & . & . & 1\end{array}$

$\begin{array}{ccccc} & 0 & 1 & 2 & 3 \\ \text { total: } & 1 & 5 & 6 & 2 \\ 0: & 1 & . & . & . \\ 1: & . & 5 & 5 & 1 \\ 2: & . & . & 1 & 1\end{array}$

$\begin{array}{cccccc} & 0 & 1 & 2 & 3 & 4 \\ \text { total: } & 1 & 5 & 9 & 7 & 2 \\ 0: & 1 & . & . & . & . \\ 1: & . & 5 & 5 & 4 & 2 \\ 2: & . & . & 4 & 3 & .\end{array}$

As in the previous example, the pruned resolution is minimal and the simplicial pruned resolution is not minimal but it is as small as possible.

Remark 4.2. It is not surprising that in the previous examples we could find a cellular minimal resolution since both the 5-path and the 5-cycle fall into [6, Example 1.7].

For larger examples the difference between the pruned and the Luybeznik resolutions becomes more apparent.

Example 4.3. Consider the ideal $I=\left\langle x_{1}^{4}, x_{2}^{4}, x_{2}^{2} x_{3}^{2}, x_{3}^{4}, x_{4}^{4}, x_{1} x_{4}^{2} x_{5}, x_{5}^{4}, x_{2}^{2} x_{6}^{2}, x_{6}^{4}, x_{4}^{2} x_{7}^{2}, x_{7}^{4}\right\rangle$. The pruned resolution is minimal while the Lyubeznik resolution is not. The Betti diagrams are:

\begin{tabular}{|c|c|c|c|c|c|c|c|c|c|c|c|c|c|c|c|c|c|c|c|c|}
\hline & & & & & & & & & & 0 & 1 & 2 & 3 & 4 & 5 & 6 & 7 & 8 & 9 & 10 \\
\hline & & & & & & & & & total: & 1 & 11 & 54 & 156 & 294 & 378 & 336 & 204 & 81 & 19 & 2 \\
\hline & 0 & 1 & 2 & 3 & 4 & 5 & 6 & 7 & $0:$ & 1 & . & . & . & . & . & . & . & . & . & . \\
\hline total: & 1 & 11 & 49 & 114 & 148 & 107 & 40 & 6 & 1: & . & . & . & . & . & . & . & . & . & . & \\
\hline $0:$ & 1 & . & . & . & . & . & . & . & 2: & . & . & . & . & . & . & . & . & & . & . \\
\hline 1: & . & . & . & . & . & . & & . & 3: & . & 11 & . & & & & . & . & . & . & . \\
\hline 2: & . & & . & . & . & . & . & . & 4: & . & . & 9 & & . & . & . & . & . & . & . \\
\hline 3: & . & 11 & & . & . & . & . & . & 5: & . & . & 2 & 7 & & . & . & . & & . & . \\
\hline 4: & . & 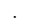 & 9 & & & & 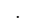 & . & 6: & . & . & 43 & 4 & 2 & . & . & . & . & . & \\
\hline 5: & . & . & 2 & 2 & . & . & . & . & 7: & . & . & . & 58 & 4 & . & . & . & . & . & . \\
\hline 6: & . & . & 38 & 4 & & . & . & . & 8: & . & . & . & 12 & 64 & 2 & . & . & . & . & . \\
\hline 7: & . & . & . & 56 & 2 & . & . & . & 9: & . & . & . & 75 & 32 & 44 & . & . & . & . & . \\
\hline 8: & . & . & . & 10 & 31 & & . & . & 10: & . & . & . & . & 106 & 48 & 22 & & . & . & 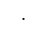 \\
\hline 9: & . & . & . & 42 & 30 & 9 & & . & 11: & . & . & . & . & 18 & 114 & 48 & 7 & & . & . \\
\hline 10: & . & & . & . & 71 & 32 & 1 & . & 12: & & & . & & 68 & 40 & 77 & 30 & 1 & & . \\
\hline 11: & . & . & . & . & 2 & 37 & 14 & . & 13: & . & . & . & . & . & 86 & 44 & 42 & 12 & & . \\
\hline 12: & . & . & . & . & 12 & 6 & 6 & 2 & 14 : & . & . & . & . & . & 10 & 85 & 30 & 18 & 2 & . \\
\hline 13: & . & & . & & & 22 & 6 & & 15: & . & . & . & . & . & 34 & 16 & 50 & 10 & 6 & \\
\hline 14: & . & . & . & . & . & & 11 & 2 & 16: & . & 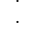 & . & . & . & . & 33 & 10 & 23 & 2 & $i$ \\
\hline 15: & . & . & . & . & . & 1 & & 1 & $17:$ & . & . & . & . & . & . & 2 & 27 & 4 & 6 & \\
\hline 16: & . & & . & . & . & . & 2 & . & 18: & . & & . & & & & 9 & 2 & 10 & & 1 \\
\hline 17: & . & . & . & . & . & . &. & 1 & 19: & . &. &. & . & . & . & . & 5 & & 3 & \\
\hline & & & & & & & & & 20: & . & . & . & . & . & . & . & & 3 & . & \\
\hline & & & & & & & & & 21: & . & $\cdot$ & & . & $\cdot$ & . & . & 1 & . & . & \\
\hline
\end{tabular}

\subsection{Independence on the characteristic of the base field}

It is well-known that the Betti numbers of a monomial ideal depend on the characteristic of the base field. This phenomenon can be understood through Hochster's formula. Namely, given a monomial ideal $I \subseteq R=\mathbb{k}\left[x_{1}, \ldots, x_{n}\right]$, we may assume that it is squarefree since its Betti numbers can always be obtained from a squarefree monomial ideal using polarization. Now, a squarefree monomial ideal $I$ is always the Stanley-Reisner ideal of a simplicial complex $\Delta$. For any squarefree degree $\alpha \in\{0,1\}^{n}$, we consider $F_{\alpha}:=\left\{x_{i} \mid \alpha_{i} \neq 0\right\} \subseteq\left\{x_{1}, \ldots, x_{n}\right\}$. Let $\Delta_{\mid F_{\alpha}}$ be the simplicial complex obtained by taking all the faces of $\Delta$ whose vertices belong to $F_{\alpha}$. Hochster's formula states that the Betti numbers of $R / I$ can be computed as the dimensions of reduced simplicial homology groups:

$$
\beta_{i, \alpha}(R / I)=\operatorname{dim}_{\mathbb{k}} \widetilde{H}_{|\alpha|-i-1}\left(\Delta_{\mid F_{\alpha}} ; \mathbb{k}\right) .
$$


By the universal coefficient theorem, we may just compute the homology modules over the integer numbers $\widetilde{H}_{|\alpha|-i-1}\left(\Delta_{\mid F_{\alpha}} ; \mathbb{Z}\right)$. It follows that Betti numbers depend on the characteristic of the base field whenever these homology groups have torsion.

Example 4.4. The most recurrent example that illustrates the behavior of Betti numbers with respect to the characteristic of the base field is the Stanley-Reisner ideal associated to a minimal triangulation of $\mathbb{P}_{\mathbb{R}}^{2}$. Namely, consider the following ideal in $R=\mathbb{k}\left[x_{1}, \ldots, x_{6}\right]$ :

$$
I=\left\langle x_{1} x_{2} x_{3}, x_{1} x_{2} x_{4}, x_{1} x_{3} x_{5}, x_{2} x_{4} x_{5}, x_{3} x_{4} x_{5}, x_{2} x_{3} x_{6}, x_{1} x_{4} x_{6}, x_{3} x_{4} x_{6}, x_{1} x_{5} x_{6}, x_{2} x_{5} x_{6}\right\rangle .
$$

The Betti diagrams in characteristic zero and two respectively are

$\begin{array}{ccccccccccc} & 0 & 1 & 2 & 3 & & 0 & 1 & 2 & 3 & 4 \\ \text { total } & 1 & 10 & 15 & 6 & \text { total: } & 1 & 10 & 15 & 7 & 1 \\ 0: & 1 & . & . & . & 0: & 1 & . & . & . & . \\ 1: & . & . & . & . & 1: & . & . & . & . & . \\ 2: & . & 10 & 15 & 6 & 2: & . & 10 & 15 & 6 & 1 \\ & & & & & 3: & . & . & . & 1 & .\end{array}$

On the other hand, the results that we obtain with the pruned and the Lyubeznik resolution respectively are:

$\begin{array}{cccccc} & 0 & 1 & 2 & 3 & 4 \\ \text { total: } & 1 & 10 & 15 & 7 & 1 \\ 0: & 1 & . & . & . & . \\ 1: & . & . & . & . & . \\ 2: & . & 10 & 15 & 6 & 1 \\ 3: & . & . & . & 1 & .\end{array}$

$\begin{array}{cccccc} & 0 & 1 & 2 & 3 & 4 \\ \text { total: } & 1 & 10 & 27 & 27 & 9 \\ 0: & 1 & . & . & . & . \\ 1: & . & . & . & . & . \\ 2: & . & 10 & 15 & 18 & 9 \\ 3: & . & . & 12 & 9 & .\end{array}$

Remark 4.5. All the free resolutions that we consider in this work are obtained from the Taylor resolution using discrete Morse theory as in [4] or [16. If one follows closely the construction of the CW-complex $X_{\mathcal{A}}$ that is homotopically equivalent to $X_{\text {Taylor }}$, one may check that it is independent of the characteristic of the base field. In this sense, the result obtained with the pruned resolution in Example 4.4 is the best that we can achieve using discrete Morse theory.

\subsection{Minimality}

A necessary and sufficient condition for the minimality of a cellular free resolution $\mathbb{F}_{\bullet}^{\left(X_{\mathcal{A}}, g r\right)}$ is described in 4, Lemma 7.5]. The pruned resolution obtained in Theorem 3.4 is minimal if and only if the following condition is satisfied: the cells $\sigma \in X_{\mathcal{A}}$ that survive to any of the pruning processes described in Section $\S 3$ satisfy $\operatorname{gr}(\sigma) \neq \operatorname{gr}\left(\sigma+\varepsilon_{j}\right)$ whenever $\sigma_{j}=0$.

Remark 4.6. Barile [3] noticed that, in the case of the Lyubeznik resolution, it is enough checking out the aforementioned condition for maximal faces of $X_{\text {Lyub }}$.

The Taylor resolution is rarely minimal. Some examples of minimal Lyubeznik resolutions include shellable ideals (see [4) and the matroid ideal of a finite projective space (see [19]). Finally, let's point out that Torrente and Varbaro 22 provided a fast algorithm for computing Betti diagrams of a monomial ideal taking as a starting point the Lyubeznik resolution of the ideal. Using the pruned resolution as starting point of their algorithm should speed up their computation of the Betti diagram.

\section{Betti splittable monomial ideals}

A technique that has been successfully applied to describe Betti numbers of monomial ideals is based on the concept of splitting introduced by Eliahou and Kervaire in [8]; see [13] for a nice survey. A refinement of this concept was coined by Francisco, Hà and Van Tuyl in [10]. 
Definition 5.1. We say that $I=J+K$ is a Betti splitting for the monomial ideal $I$ if the following formula for the $\mathbb{Z}^{n}$-graded Betti numbers is satisfied:

$$
\beta_{i, \alpha}(I)=\beta_{i, \alpha}(J)+\beta_{i, \alpha}(K)+\beta_{i-1, \alpha}(J \cap K) .
$$

We can mimic the same concept introducing the pruned Betti numbers as the Betti numbers that we obtain in the pruned free resolution of Section 3.1. and that we will denote by $\bar{\beta}_{i, \alpha}(I)$ to distinguish them from the usual Betti numbers.

Definition 5.2. We say that $I=J+K$ is a pruned Betti splitting for the monomial ideal $I$ if the following formula for the $\mathbb{Z}^{n}$-graded Betti numbers is satisfied:

$$
\bar{\beta}_{i, \alpha}(I)=\bar{\beta}_{i, \alpha}(J)+\bar{\beta}_{i, \alpha}(K)+\bar{\beta}_{i-1, \alpha}(J \cap K) .
$$

Remark 5.3. Obviously, a pruned Betti splitting provides a Betti splitting whenever the pruning algorithm gives a minimal free resolution of $I$.

Necessary and sufficient conditions describing Betti splittings have been given in [10]. Using our main Algorithm 3.1, we will give some conditions that are easy to check and that provide a pruned Betti splitting.

\subsection{A partial pruning of $J \cap K$}

Let $I=\left\langle m_{1}, \ldots, m_{s+r}\right\rangle$ be a monomial ideal, and consider the Taylor simplicial complex $X_{\text {Taylor }}$ on $r$ vertices which faces are labeled by $\sigma \in\{0,1\}^{s+r}$. Consider a decomposition $I=J+K$ with $J=\left\langle m_{1}, \ldots, m_{s}\right\rangle$ and $K=\left\langle m_{s+1}, \ldots, m_{s+r}\right\rangle$. In order to compute the pruned resolution of $J$ and $K$ we have to consider the subcomplexes:

- $X_{J} \subseteq X_{\text {Taylor }}$ with faces labelled by $\sigma=\left(\sigma_{1}, \ldots, \sigma_{s}, 0, \ldots, 0\right) \in\{0,1\}^{s+r}$, and

- $X_{K} \subseteq X_{\text {Taylor }}$ with faces labelled by $\sigma=\left(0, \ldots, 0, \sigma_{s+1}, \ldots, \sigma_{s+r}\right) \in\{0,1\}^{s+r}$.

Denote by $X^{\prime}$ the set obtained by removing the faces of $X_{J}$ and $X_{K}$ from $X_{\text {Taylor }}$. Notice that $X^{\prime}$ is not a simplicial subcomplex of $X_{\text {Taylor }}$ and, in particular, it is not the Taylor simplicial complex associated to the intersection. However, applying a partial pruning algorithm to the Taylor complex $X_{J \cap K}$ associated to a (possibly non-minimal) set of generators of $J \cap K$ we obtain $X^{\prime}$. The proof of this fact is quite tedious but straightforward. We will simply sketch it and leave the details for the interested reader.

Consider the (possibly non-minimal) set of generators of $J \cap K$ given by the monomials

$$
\left\{m_{1, s+1}, \ldots, m_{s, s+1}, m_{1, s+2}, \ldots, m_{s, s+2}, \ldots, m_{1, r}, \ldots, m_{s, s+r}\right\}
$$

where $m_{i, s+1+k}=\operatorname{lcm}\left(m_{i}, m_{s+1+k}\right)$. In what follows we will also denote $m_{i_{1}, \ldots, i_{\ell}}=\operatorname{lcm}\left(m_{i_{1}}, \ldots, m_{i_{\ell}}\right)$. The Taylor complex $X_{J \cap K}$ is the full simplicial complex on $s r$ vertices with faces labelled by $\sigma \in\{0,1\}^{s r}$. Notice that the vertex $\varepsilon_{j}$ corresponds to the monomial $m_{\varepsilon_{j}}:=m_{i, s+1+k}$ if we have $j=k s+i$ for $k \in\{0, \ldots, r-1\}$ and $i \in\{1, \ldots, s\}$. In general, a face $\sigma$ corresponds to $m_{\sigma}:=\operatorname{lcm}\left(m_{\varepsilon_{j}} \mid \sigma_{j}=1\right)$.

Now we want to apply our Algorithm 3.1 but we only want to prune the edges $\sigma+\varepsilon_{j} \longrightarrow \sigma$ whenever the corresponding $\mathrm{lcm}$ 's involve the same monomials $m_{i} \in I$. For example, we have that $\mathrm{lcm}\left(m_{i, b}, m_{a, s+1+k}\right)=$ $\operatorname{lcm}\left(m_{i, s+1+k}, m_{i, b}, m_{a, s+1+k}\right)=m_{i, s+1+k, a, b}$ so both lcm 's involve the same monomials $m_{i}, m_{s+1+k}, m_{a}, m_{b} \in$ $I$ for all $a, b$. This means that the corresponding edge would be pruned at step $j=k s+i$.

The partial pruning algorithm that we apply is the following: 


\section{Algorithm 5.4. (Partial pruning)}

InPUT: The set of edges $E_{X_{J \cap K}}$.

For $j$ from 1 to $s r$, incrementing by 1

(j) Prune the edge $\sigma+\varepsilon_{j} \longrightarrow \sigma$ for all $\sigma \in\{0,1\}^{s r}$ such that $\sigma_{j}=0$, where 'prune' means remove the edge if it survived after step $(j-1)$ and $m_{\sigma}$ involves the monomials $m_{i}$ and $m_{s+1+k}$, where $j=k s+i$.

RETURN: The set $\mathcal{A}^{\prime}$ of edges that have been pruned.

The tedious part is to check that using this algorithm we obtain $X^{\prime}$, that is, $X_{\mathcal{A}^{\prime}}=X^{\prime}$. To illustrate this fact we present the following:

Example 5.5. Let $I=\left\langle m_{1}, m_{2}, m_{3}, m_{4}\right\rangle$ be a monomial ideal generated by four monomials. We are going to consider the following decompositions:

- Consider $I=J+K$ with $J=\left\langle m_{1}, m_{2}, m_{3}\right\rangle$ and $K=\left\langle m_{4}\right\rangle$. In this case we have $J \cap K=$ $\left\langle m_{1,4}, m_{2,4}, m_{3,4}\right\rangle$ so $X^{\prime}$ coincides with $X_{J \cap K}$.

. If $J=\left\langle m_{1}, m_{2}\right\rangle$ and $K=\left\langle m_{3}, m_{4}\right\rangle$ then we have that $J \cap K=\left\langle m_{1,3}, m_{2,3}, m_{1,4}, m_{2,4}\right\rangle$. The directed graph associated to the Taylor simplicial complex of $I$ is:

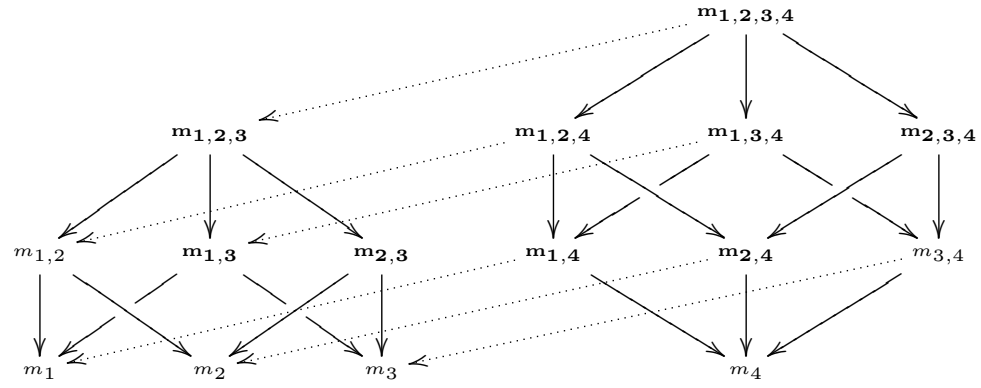

and the monomials corresponding to the set $X^{\prime}$ are indicated with bold letters. On the other hand, applying the partial pruning algorithm to the Taylor complex $X_{J \cap K}$ we also get the set $X^{\prime}$ as shown in the following graph:

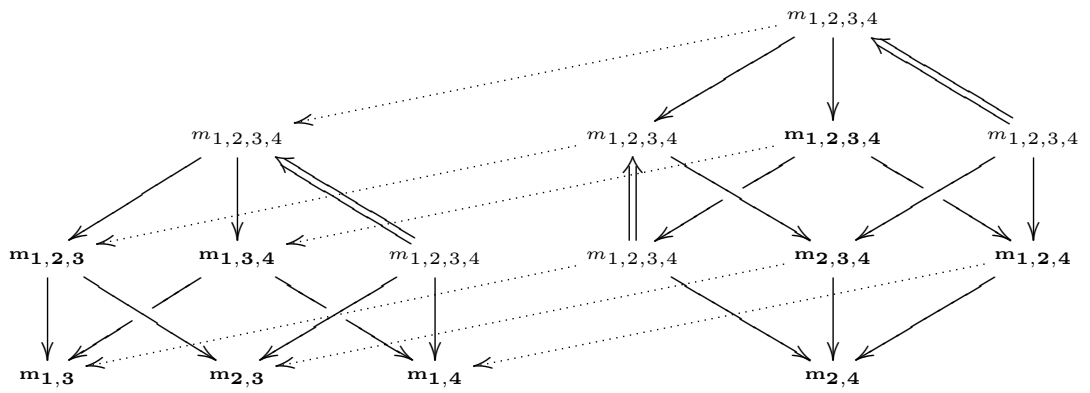

\subsection{A sufficient condition for Betti splittings}

Let $I=J+K$ be a decomposition of a monomial ideal. In order to have a Betti splitting, it is enough to check that applying Algorithm 3.1 to $I$, the pruning steps are realized independently within the edges associated to $X_{J}, X_{K}$ and $X^{\prime}$. Notice that in this case we obtain the pruned Betti numbers of $I$ from 
the pruned Betti numbers of $J, K$ and $J \cap K$ and thus we get a sufficient condition for a Betti splitting. We collect this fact in the following:

Proposition 5.6. Let $I=\left\langle m_{1}, \ldots, m_{s+r}\right\rangle \subseteq \mathbb{k}\left[x_{1}, \ldots, x_{n}\right]$ be a monomial ideal and consider the ideals $J=\left\langle m_{1}, \ldots, m_{s}\right\rangle$ and $K=\left\langle m_{s+1}, \ldots, m_{s+r}\right\rangle$. If we only perform pruning steps within the edges of $X_{J}$, $X_{K}$ or $X^{\prime}$ separately in Algorithm 3.1, then $I=J+K$ is a pruned Betti splitting for $I$.

In the particular case that we remove just one generator from our initial ideal, we obtain the following:

Corollary 5.7. Let $I=\left\langle m_{1}, \ldots, m_{s+1}\right\rangle \subseteq \mathbb{k}\left[x_{1}, \ldots, x_{n}\right]$ be a monomial ideal and consider the ideals $J=\left\langle m_{1}, \ldots, m_{s}\right\rangle$ and $K=\left\langle m_{s+1}\right\rangle$. If no pruning has been made at the step $s+1$ of Algorithm 3.1, then $I=J+K$ is a pruned Betti splitting for $I$.

Betti splitting techniques can be used to provide a recursive method to compute Betti numbers of monomial ideals. For example, the case of edge ideals of graphs has been successfully studied in 12 ] and 10 where they considered splitting edges and splitting vertices. Splitting edges are easy to describe (see [10. Theorem 3.4]) but, in general they are difficult to find. On the other hand, every vertex is a splitting vertex except for some limit cases where the vertex is isolated or its complement consists of isolated vertices.

One can also check that any vertex also provides a pruned Betti splitting. Indeed, let $I=I(G) \subseteq$ $\mathbb{k}\left[x_{1}, \ldots, x_{n}\right]$ be the edge ideal of a graph $G$ on $n$ vertices. Consider a vertex, say $x_{n}$, and the decomposition $I=J+K$ where $J=I\left(G \backslash\left\{x_{n}\right\}\right)$ is the edge ideal of the graph obtained from $G$ by removing the vertex $x_{n}$ and all the edges passing through this vertex. Notice that $K$ is the edge ideal of a bipartite graph $\mathcal{K}_{1, d}$ where $d$ is the number of vertices adjacent to $x_{n}$ in $G$. We have a pruned Betti splitting because the ideal $J$ does not involve the variable $x_{n}$ and the pruning steps of the algorithm are performed within $X_{K}$ and $X^{\prime}$ separately. Moreover, it is not difficult to see that the pruning algorithm provides a minimal free resolution for $K$, and hence

$$
\bar{\beta}_{i, \alpha}(I)=\bar{\beta}_{i, \alpha}(J)+\beta_{i, \alpha}(K)+\bar{\beta}_{i-1, \alpha}(J \cap K) .
$$

In particular, if Algorithm 3.1 provides a minimal free resolution for $J$ and $J \cap K$, then it is also provides a minimal free resolution for $I$.

For paths and cycles, we can use these splitting techniques to prove that the pruning algorithm will always provide a minimal free resolution as we already observed in Example 4.1 for the 5-path and the 5 -cycle. The Betti numbers of these ideals have already been computed by Jacques in [15.

Example 5.8 ( $n$-paths). Let $I=\left\langle x_{1} x_{2}, x_{2} x_{3}, \ldots, x_{n-1} x_{n}\right\rangle$ be the edge ideal of an $n$-path. A decomposition $I=J+K$ with $J=\left\langle x_{1} x_{2}, x_{2} x_{3}, \ldots, x_{n-2} x_{n-1}\right\rangle$ and $K=\left\langle x_{n-1} x_{n}\right\rangle$ is a pruned Betti splitting simply because the ideal $J$ does not involve the vertex $x_{n}$. Therefore we have

$$
\bar{\beta}_{i, \alpha}(I)=\bar{\beta}_{i, \alpha}(J)+\bar{\beta}_{i, \alpha}(K)+\bar{\beta}_{i-1, \alpha}(J \cap K) .
$$

Indeed, this is a Betti splitting and the pruning algorithm provides a minimal free resolution, thus

$$
\beta_{i, \alpha}(I)=\beta_{i, \alpha}(J)+\beta_{i, \alpha}(K)+\beta_{i-1, \alpha}(J \cap K) .
$$

By induction, we get a minimal free resolution for the ideals $J$ and $K$ so we only have to control the intersection in order to get the desired result. Recall that, using Lemma 3.6, we only have to consider a minimal set of generators. In our case we have

$$
J \cap K=\langle\underbrace{x_{1} x_{2} x_{n-1} x_{n}, \ldots, x_{n-4} x_{n-3} x_{n-1} x_{n}}_{J^{\prime}}, \underbrace{x_{n-2} x_{n-1} x_{n}}_{K^{\prime}}\rangle .
$$


If we consider the decomposition $J \cap K=J^{\prime}+K^{\prime}$, we have a Betti splitting. Namely, the pruning algorithm applied on the ideals $J^{\prime}$ and $J^{\prime} \cap K^{\prime}$ is equivalent to the one given for the path $\left\langle x_{1} x_{2}, \ldots, x_{n-4} x_{n-3}\right\rangle$, and we are done by induction.

Example 5.9 ( $n$-cycles). Let $I=\left\langle x_{1} x_{2}, x_{2} x_{3}, \ldots, x_{n-1} x_{n}, x_{n} x_{1}\right\rangle$ be the edge ideal of an $n$-cycle. The decomposition $I=J+K$ with $J=\left\langle x_{1} x_{2}, x_{2} x_{3}, \ldots, x_{n-1} x_{n}\right\rangle$ and $K=\left\langle x_{n} x_{1}\right\rangle$ is not a Betti splitting by 10, Theorem 3.4]. Indeed, there is a pruning in the last step of Algorithm 3.1 .

It is more convenient to consider a splitting vertex. Namely, consider $I=J+K$ with $J=$ $\left\langle x_{1} x_{2}, x_{2} x_{3}, \ldots, x_{n-2} x_{n-1}\right\rangle$ and $K=\left\langle x_{n-1} x_{n}, x_{n} x_{1}\right\rangle$. Since $J$ and $K$ are the ideal of an $(n-1)$-path and a 3-path respectively, the pruning algorithm provides a minimal free resolution as shown in Example 5.8 . Therefore we have:

$$
\bar{\beta}_{i, \alpha}(I)=\beta_{i, \alpha}(J)+\beta_{i, \alpha}(K)+\bar{\beta}_{i-1, \alpha}(J \cap K) .
$$

A minimal set of generators of $J \cap K$ is

$$
\langle\underbrace{x_{2} x_{3} x_{n-1} x_{n}, \ldots, x_{n-4} x_{n-3} x_{n-1} x_{n}, x_{n-2} x_{n-1} x_{n}}_{J^{\prime}}, \underbrace{x_{1} x_{2} x_{n}, x_{1} x_{3} x_{4} x_{n}, \ldots, x_{1} x_{n-3} x_{n-2} x_{n}}_{K^{\prime}}\rangle .
$$

We have a pruned Betti splitting given by $J \cap K=J^{\prime}+K^{\prime}$. The pruning algorithm gives a minimal free resolution for the ideals $J^{\prime}$ and $K^{\prime}$ as we have seen when dealing with the case of paths. A minimal set of generators for the intersection $J^{\prime} \cap K^{\prime}$ is

$$
\left\langle x_{1} x_{2} x_{3} x_{n-1} x_{n}, x_{1} x_{3} x_{4} x_{n-1} x_{n}, \ldots, x_{1} x_{n-3} x_{n-2} x_{n-1} x_{n}, x_{1} x_{2} x_{n-2} x_{n-1} x_{n}\right\rangle
$$

but the pruning algorithm applied to this ideal is equivalent to the one for the cycle $\left\langle x_{2} x_{3}, x_{3} x_{4}, \ldots\right.$, $\left.x_{n-3} x_{n-2}, x_{2} x_{n-2}\right\rangle$, and we are done by induction.

\section{Acknowledgement}

We would like to thank the referees for their meticulous comments that helped us to improve this manuscript.

\section{References}

[1] J. Abbot and A. M. Bigatti, CoCoALib: a C++ library for doing Computations in Commutative Algebra, available at http://cocoa.dima.unige.it/cocoalib.

[2] J.Àlvarez Montaner and K. Yanagawa, Lyubeznik numbers of local rings and linear strands of graded ideals, Nagoya Math. J. 231 (2019), 23-54.

[3] M. Barile, On ideals whose radical is a monomial ideal, Comm. Alg. 33 (2005), 4479-4490.

[4] E. Batzies and V. Welker, Discrete Morse theory for cellular resolutions, J. Reine Angew. Math. 543 (2002), 147-168.

[5] D. Bayer, I. Peeva and B. Sturmfels, Monomial resolutions, Math. Res. Lett. 5 (1998), 31-46.

[6] D. Bayer and B. Sturmfels, Cellular resolutions of monomial modules, J. Reine Angew. Math. 502 (1998), 123-140.

[7] M. K. Chari, On discrete Morse functions and combinatorial decompositions, Discrete Math. 217 (2000), 101-113. 
[8] S. Eliahou and M. Kervaire, Minimal resolutions of some monomial ideals, J. Algebra 129 (1990), $1-25$.

[9] R. Forman, Morse theory for cell complexes, Adv. Math. 134 (1998), 90-145.

[10] C. Francisco, H. T. Hà and A. Van Tuyl, Splittings of monomial ideals, Proc. Amer. Math. Soc. 137 (2009), 3271-3282.

[11] V. Gasharov, I. Peeva, and V. Welker, The lcm-lattice in monomial resolutions, Math. Res. Lett. 6 (1999), 521-532.

[12] H. T. Hà and A. Van Tuyl, Splittable ideals and the resolution of monomial ideals, J. Algebra 309 (2007), 405-425.

[13] H. T. Hà and A. Van Tuyl, Resolutions of squarefree monomial ideals via facet ideals: a survey, Contemp. Math. 441 (2007), 91-117.

[14] M. Hochster, Cohen-Macaulay rings, combinatorics, and simplicial complexes, Ring theory, II (Proc. Second Conf., Univ. Oklahoma, Norman, Okla., 1975), pp. 171-223. Lecture Notes in Pure and Appl. Math., Vol. 26, Dekker, New York, 1977.

[15] S. Jacques, Betti Numbers of Graph Ideals, Ph.D. Thesis, Univ. of Sheffield (2004), available at arXiv:math/0410107.

[16] M. Jöllenbeck and V. Welker, Minimal resolutions via algebraic discrete Morse theory, Mem. Amer. Math. Soc. 197 (2009).

[17] G. Lyubeznik, A new explicit finite free resolution of ideals generated by monomials in an $R$-sequence, J. Pure and Appl. Algebra 51 (1988), 193-195.

[18] E. Miller, B. Sturmfels and K. Yanagawa, Generic and cogeneric monomial ideals, J. Symbolic Comput. 29 (2000), 691-708.

[19] I. Novik, Lyubeznik's resolution and rooted complexes, J. Algebraic Comb. 16 (2002), 97-101.

[20] E. Sköldberg, Morse theory from an algebraic viewpoint, Trans. Amer. Math. Soc. 358 (2006), $115-129$.

[21] D. Taylor, Ideals generated by an R-sequence, PhD-Thesis, University of Chicago, 1966.

[22] M-L Torrente and M. Varbaro, Computing the Betti table of a monomial ideal: a reduction algorithm, J. Symbolic Comput. 87 (2018), 87-98.

[23] M. Velasco, Minimal free resolutions that are not supported by a $C W$-complex, J. Algebra 319 (2008), 102-114.

[24] V. Welker, Discrete Morse theory and free resolutions, in: Algebraic Combinatorics, Universitext, Springer, Berlin (2007), 81-172. 